\title{
Self-consistent approach for Bose-condensed atoms in optical lattices
}

\author{
V.I. Yukalov \\ Bogolubov Laboratory of Theoretical Physics, Joint Institute for Nuclear Research, 141980 Dubna, Russia \\ Received December 7, 2012, in final form March 6, 2013 \\ Bose atoms in optical lattices are considered at low temperatures and weak interactions, when Bose-Einstein \\ condensate is formed. A self-consistent approach, based on the use of a representative statistical ensemble, is \\ employed, ensuring a gapless spectrum of collective excitations and the validity of conservation laws. In order \\ to show that the approach is applicable to both weak and tight binding, the problem is treated in the Bloch as \\ well as in the Wannier representations. Both these ways result in similar expressions that are compared for the \\ self-consistent Hartree-Fock-Bogolubov approximation. A convenient general formula for the superfluid fraction \\ of atoms in an optical lattice is derived.
}

Key words: Bose-Einstein condensate, representative ensemble, optical lattices, Bloch representation, Wannier representation, superfluidity

PACS: 03.75. Hh, 03.75.Nt, 05.30.Ch, 05.30.Jp, 05.70.Ce

\section{Introduction}

Systems with Bose-Einstein condensate are interesting objects from both theoretical and experimental points of view. That is why they have been intensively studied in recent years. Vast literature on this problem can be found in the books [1-4] and review articles [5-15]. Creation of optical lattices has made it possible to achieve a new dimension in the physics of cold atoms, providing an opportunity for numerous novel applications and for modeling many effects typical of condensed matter [16-19].

The occurrence of Bose-Einstein condensate is intimately related to the global gauge symmetry breaking [2, 11] that is a necessary and sufficient condition for Bose-Einstein condensation. In the theory of Bose-condensed systems, there exists an old problem, formulated by Hohenberg and Martin [20], who showed that, as soon as gauge symmetry is broken, the description of such a system suffers from one of the defects, either yielding unphysical spectrum of collective excitations or resulting in broken conservation laws and incorrect thermodynamics. Any of these deficiencies implies that the description is not self-consistent, corresponding to an unstable system. This problem has been solved by employing representative statistical ensembles [21-23] to systems with a broken gauge symmetry [24-27]. This approach was shown to be completely self-consistent and gapless, with the Hartree-Fock-Bogolubov (HFB) approximation [28, 29] providing an accurate description for uniform Bose systems [27, 30-32], as well as for these systems in random external potentials [33,34].

In the present paper, this self-consistent approach is applied to Bose-condensed atoms in optical lattices. Sections 2 and 3, contain the main definitions related to optical lattices and Bose-condensed atoms, respectively. In section 4, the Bloch representation is used, which can be more suitable for weak binding, while in section 5 , the Wannier representation is employed, which is more convenient for tight binding. Both these cases are treated in the HFB approximation leading to similar results. However, the Wannier representation, yielding the Hubbard Hamiltonian, is a bit simpler. Some thermodynamic characteristics are considered in section 6, where a general and convenient formula for superfluid fraction is derived. Section 7 concludes. 
Throughout the paper, the system of units is used, where the Planck and Boltzmann constants are set to be one.

\section{Optical lattices}

Optical lattices are created by laser beams forming standing waves, which corresponds to the formation of a periodic lattice potential

$$
V_{\mathrm{L}}(\mathbf{r}+\mathbf{a})=V_{\mathrm{L}}(\mathbf{r})
$$

with a being a lattice vector with the components $a_{\alpha}=\lambda_{\alpha} / 2$, where $\lambda_{\alpha}$ is a laser wavelength and $\alpha=1,2, \ldots, d$ enumerates spatial components in a $d$-dimensional space. The standard form of the lattice potential is

$$
V_{\mathrm{L}}(\mathbf{r})=\sum_{\alpha=1}^{d} V_{\alpha} \sin ^{2}\left(k_{0}^{\alpha} r_{\alpha}\right)
$$

with the laser wave vector

$$
\mathbf{k}_{0}=\left\{k_{0}^{\alpha}=\frac{2 \pi}{\lambda_{\alpha}}=\frac{\pi}{a_{\alpha}}\right\} .
$$

The lattice depth is defined by the parameter

$$
V_{0} \equiv \frac{1}{d} \sum_{\alpha=1}^{d} V_{\alpha} .
$$

Another important quantity, characterizing an optical lattice, is the recoil energy

$$
E_{\mathrm{R}} \equiv \frac{k_{0}^{2}}{2 m}, \quad k_{0}^{2} \equiv \sum_{\alpha=1}^{d}\left(k_{0}^{\alpha}\right)^{2},
$$

where $m$ is atomic mass. The ratio $E_{\mathrm{R}} / V_{0}$ characterizes the relative lattice depth.

\section{Bose atoms}

The lattice is loaded with Bose atoms, whose interactions are measured by means of the scattering length $a_{\mathrm{s}}$ entering the effective interaction strength

$$
\Phi_{0} \equiv 4 \pi \frac{a_{\mathrm{s}}}{m} .
$$

The energy operator is given by the Hamiltonian

$$
\hat{H}=\int \hat{\psi}^{\dagger}(\mathbf{r})\left(-\frac{\nabla^{2}}{2 m}+U+V_{\mathrm{L}}\right) \hat{\psi}(\mathbf{r}) \mathrm{d} \mathbf{r}+\frac{1}{2} \Phi_{0} \int \hat{\psi}^{\dagger}(\mathbf{r}) \hat{\psi}^{\dagger}(\mathbf{r}) \hat{\psi}(\mathbf{r}) \hat{\psi}(\mathbf{r}) \mathrm{d} \mathbf{r},
$$

in which $U=U(\mathbf{r})$ is a trapping potential, if any, and $V_{\mathrm{L}}=V_{\mathrm{L}}(\mathbf{r})$ is a lattice potential. The atom field operators $\hat{\psi}(\mathbf{r})$ satisfy the Bose commutation relations.

The existence of Bose-Einstein condensate necessarily requires that global gauge symmetry should be broken [2, 11]. The most straightforward way of the gauge symmetry breaking is by means of the Bogolubov shift of the field operator

$$
\hat{\psi}(\mathbf{r})=\eta(\mathbf{r})+\psi_{1}(\mathbf{r})
$$

Here, the first term is the condensate wave function normalized to the number of condensed atoms

$$
N_{0}=\int|\eta(\mathbf{r})|^{2} d \mathbf{r} .
$$


The second term is the field operator of uncondensed atoms, whose number is given by the statistical average

$$
N_{1}=\left\langle\hat{N}_{1}\right\rangle, \quad \hat{N}_{1} \equiv \int \psi_{1}^{\dagger}(\mathbf{r}) \psi_{1}(\mathbf{r}) \mathrm{d} \mathbf{r}
$$

of the number-of-particle operator $\hat{N}_{1}$.

The uncondensed atoms are normal in the sense that the average of their field operator is zero,

$$
\left\langle\psi_{1}\right\rangle=0 .
$$

To avoid double counting of the degrees of freedom, the orthogonality condition

$$
\int \eta^{*}(\mathbf{r}) \psi_{1}(\mathbf{r}) \mathrm{d} \mathbf{r}=0
$$

is required. This condition is a direct consequence of orthogonality of wave functions serving as a basis for the expansion of the field operator $\hat{\psi}(\mathbf{r})[28,29]$.

The number of atoms per lattice site is called a filling factor that is defined as the ratio

$$
v \equiv \frac{N}{N_{\mathrm{L}}}=\rho a^{d} \quad\left(N=N_{0}+N_{1}\right),
$$

in which $a$ is a mean interatomic distance and $\rho$ is the average atomic density,

$$
a \equiv\left(\frac{V}{N_{\mathrm{L}}}\right)^{1 / d}, \quad \rho \equiv \frac{N}{V} .
$$

The representative ensemble for a system with a broken gauge symmetry is characterized [24-27] by the grand Hamiltonian

$$
H=\hat{H}-\mu_{0} N_{0}-\mu_{1} \hat{N}_{1}-\hat{\Lambda},
$$

where $\mu_{0}$ and $\mu_{1}$ are the Lagrange multipliers ensuring the validity of normalizations (3.4) and (3.5), while the term $\hat{\Lambda}$ is defined so that the terms linear in the operators $\psi_{1}$ are cancelled in the Hamiltonian, which ensures the condition (3.6).

It is worth stressing that the introduction of two Lagrange multipliers, $\mu_{0}$ and $\mu_{1}$ is necessary due to the presence of two independent variables in the Bogolubov shift (3.3) and the related two normalization conditions (3.4) and (3.5). It is a general mathematical fact that the number of Lagrange multipliers should be equal to the number of imposed constraints, such as the normalization conditions. The theory can become non-self-consistent if the number of Lagrange multipliers is smaller than that of the imposed constraints. Introducing two Lagrange multipliers does not exclude that in particular cases, these multipliers could become equal, as it happens in the Bogolubov approximation [28, 29]. The physical meaning of using two Lagrange multipliers has been thoroughly explained in the previous papers \11, 14, 19, 2327, 30-33].

\section{Bloch representation}

One usually considers optical lattices by reducing the problem to a Hubbard Hamiltonian by means of the Wannier representation which is convenient in the case of a tight binding. Here, we show that it is equivalently possible to employ the Bloch representation that can be more appropriate for weak binding and leads to the results similar to those in the Wannier representation to be considered in the following section. Below, we assume that there is no trapping potential, so that the system is ideally periodic.

Let $\left\{\varphi_{n k}(\mathbf{r})\right\}$ be the basis of Bloch functions labeled by the zone index $n$ and quasi-momentum multiindex $k$. Then, the field operators of uncondensed atoms can be expanded over this basis,

$$
\psi_{1}(\mathbf{r})=\sum_{n k} a_{n k} \varphi_{n k}(\mathbf{r})
$$


The basis should be chosen so that the Bloch functions are natural orbitals [35], that is, the eigenfunctions of the density matrix

$$
\rho_{1}\left(\mathbf{r}, \mathbf{r}^{\prime}\right) \equiv\left\langle\psi_{1}^{\dagger}\left(\mathbf{r}^{\prime}\right) \psi_{1}(\mathbf{r})\right\rangle .
$$

Then, the density matrix enjoys a diagonal expansion

$$
\rho_{1}\left(\mathbf{r}, \mathbf{r}^{\prime}\right)=\sum_{n k}\left\langle a_{n k}^{\dagger} a_{n k}\right\rangle \varphi_{n k}(\mathbf{r}) \varphi_{n k}^{*}\left(\mathbf{r}^{\prime}\right) .
$$

In other words, the use of natural orbitals simplifies the consideration due to the following properties

$$
\left\langle a_{n k}^{\dagger} a_{m p}\right\rangle=\delta_{m n} \delta_{k p}\left\langle a_{n k}^{\dagger} a_{n k}\right\rangle, \quad\left\langle a_{n k} a_{m p}\right\rangle=\delta_{m n} \delta_{-k p}\left\langle a_{n k} a_{n p}\right\rangle .
$$

Substituting expansion (4.1) into the grand Hamiltonian (3.10) gives the sum

$$
H=H^{(0)}+H^{(2)}+H^{(3)}+H^{(4)}
$$

Here, the first term

$$
H^{(0)}=\int \eta^{*}(\mathbf{r})\left(-\frac{\nabla^{2}}{2 m}+V_{\mathrm{L}}-\mu_{0}\right) \eta(\mathbf{r}) \mathrm{d} \mathbf{r}+\frac{1}{2} \Phi_{0} \int|\eta(\mathbf{r})|^{4} \mathrm{~d} \mathbf{r}
$$

contains only a condensate wave function, but no field operators of uncondensed atoms. The term, linear in $\psi_{1}$, is canceled by the Lagrange term $\hat{\Lambda}$. In the following expressions, the pair $\{n, k\}$, for brevity, will be denoted as $k$, while the set $\{n,-k\}$, as $-k$. Then, the term, containing the products of two operators of uncondensed atoms, reads as

$$
\begin{aligned}
H^{(2)}= & \sum_{k p}\left[\int \varphi_{k}^{*}(\mathbf{r})\left(-\frac{\nabla^{2}}{2 m}+V_{\mathrm{L}}-\mu_{1}+2 \Phi_{0}|\eta(\mathbf{r})|^{2}\right) \varphi_{p}(\mathbf{r}) \mathrm{d} \mathbf{r}\right] a_{k}^{\dagger} a_{p} \\
& +\frac{1}{2} \sum_{k p}\left(\Phi_{k p} a_{k}^{\dagger} a_{p}^{\dagger}+\Phi_{k p}^{*} a_{p} a_{k}\right)
\end{aligned}
$$

where

$$
\Phi_{k p} \equiv \Phi_{0} \int \varphi_{k}^{*}(\mathbf{r}) \varphi_{p}^{*}(\mathbf{r}) \eta^{2}(\mathbf{r}) \mathrm{d} \mathbf{r} .
$$

The term of third order, with respect to the products of the field operators of uncondensed atoms, is

$$
H^{(3)}=\sum_{k p q}\left(\int \Phi_{k p q} a_{k}^{\dagger} a_{p}^{\dagger} a_{q}+\Phi_{k p q}^{*} a_{q}^{\dagger} a_{p} a_{k}\right)
$$

with

And the fourth-order term is

$$
\Phi_{k p q} \equiv \Phi_{0} \int \varphi_{k}^{*}(\mathbf{r}) \varphi_{p}^{*}(\mathbf{r}) \varphi_{q}(\mathbf{r}) \eta(\mathbf{r}) \mathrm{d} \mathbf{r}
$$

$$
H^{(4)}=\frac{1}{2} \sum_{k p q l} \Phi_{k p q l} a_{k}^{\dagger} a_{p}^{\dagger} a_{q} a_{l}
$$

where

$$
\Phi_{k p q l} \equiv \Phi_{0} \int \varphi_{k}^{*}(\mathbf{r}) \varphi_{p}^{*}(\mathbf{r}) \varphi_{q}(\mathbf{r}) \varphi_{l}(\mathbf{r}) \mathrm{d} \mathbf{r} .
$$

In the Hartree-Fock-Bogolubov (HFB) approximation, the third-order term $H^{(3)}$ yields expressions linear in $\psi_{1}$, which should be canceled by the Lagrange canceler $\hat{\Lambda}$. The fourth-order part takes the form

$$
\begin{aligned}
H^{(4)}= & \frac{1}{2} \sum_{k p q}\left(4 \Phi_{k q q p} n_{q} a_{k}^{\dagger} a_{p}+\Phi_{k p q q} \sigma_{q} a_{k}^{\dagger} a_{p}^{\dagger}+\Phi_{k p q q}^{*} \sigma_{q}^{*} a_{p} a_{k}\right) \\
& -\frac{1}{2} \sum_{k p}\left(2 \Phi_{k p p k} n_{k} n_{p}+\Phi_{k k p p} \sigma_{k}^{*} \sigma_{p}\right),
\end{aligned}
$$


in which the notations for the so-called normal

$$
n_{k} \equiv\left\langle a_{k}^{\dagger} a_{k}\right\rangle,
$$

and anomalous

$$
\sigma_{k} \equiv\left\langle a_{k} a_{-k}\right\rangle
$$

averages are used. The normal average (4.11) is the distribution of atoms, while the absolute value $\left|\sigma_{k}\right|$ of the anomalous average (4.12) is the distribution of the correlated atomic pairs [19, 25, 29].

Let us introduce the notation

$$
\omega_{k p} \equiv \int \varphi_{k}^{*}(\mathbf{r})\left(-\frac{\nabla^{2}}{2 m}+V_{\mathrm{L}}+2 \Phi_{0}|\eta|^{2}\right) \varphi_{p}(\mathbf{r}) \mathrm{d} \mathbf{r}+2 \sum_{q} \Phi_{k q q p} n_{q}-\mu_{1} \delta_{k p}
$$

and

$$
\Delta_{k p} \equiv \Phi_{k p}+\sum_{q} \Phi_{k p q q} \sigma_{q} .
$$

Then, the grand Hamiltonian (4.5) in the HFB approximation can be written as

$$
H=E_{\mathrm{HFB}}+\sum_{k p} \omega_{k p} a_{k}^{\dagger} a_{p}+\frac{1}{2} \sum_{k p}\left(\Delta_{k p} a_{k}^{\dagger} a_{p}^{\dagger}+\Delta_{k p}^{*} a_{p} a_{k}\right),
$$

where the first term is the nonoperator quantity

$$
E_{\mathrm{HFB}}=H^{(0)}-\frac{1}{2} \sum_{k p}\left(2 \Phi_{k p p k} n_{k} n_{p}+\Phi_{k k p p} \sigma_{k}^{*} \sigma_{p}\right) .
$$

The quadratic Hamiltonian (4.15) can be diagonalized and all observables calculated. However, the resulting expressions are rather complicated. In order to simplify the calculations, it is possible to assume that the main contribution in the above formulas comes from diagonal terms, since the Bloch functions are mutually orthogonal. This can be referred to as the diagonal approximation, when expressions (4.13) and (4.14) take the form

$$
\omega_{k p}=\delta_{k p} \omega_{k}, \quad \Delta_{k p}=\delta_{-k p} \Delta_{k},
$$

in which

$$
\omega_{k}=\int \varphi_{k}^{*}(\mathbf{r})\left(-\frac{\nabla^{2}}{2 m}+V_{\mathrm{L}}+2 \Phi_{0}|\eta(\mathbf{r})|^{2}\right) \varphi_{k}(\mathbf{r}) \mathrm{d} \mathbf{r}+2 \sum_{q} \Phi_{k q q k} n_{q}-\mu_{1}
$$

and

$$
\Delta_{k}=\Phi_{-k k}+\sum_{q} \Phi_{-k k q q} \sigma_{q}
$$

The use of the diagonal approximation is not compulsory and it is possible to diagonalize the quadratic form (4.15) without it. This approximation, however, essentially simplifies the formulas. Justification of this approximation is based on the fact that the expansion functions $\varphi_{k}$ are mutually orthogonal, which makes it reasonable to assume that the matrix elements over these functions are such that their diagonal elements are larger than off-diagonal.

In the diagonal approximation, Hamiltonian (4.15) reduces to

$$
H=E_{\mathrm{HFB}}+\sum_{k} \omega_{k} a_{k}^{\dagger} a_{k}+\frac{1}{2} \sum_{k}\left(\Delta_{k} a_{k}^{\dagger} a_{-k}^{\dagger}+\Delta_{k}^{*} a_{-k} a_{k}\right) .
$$

This form is much simpler to diagonalize using the Bogolubov canonical transformation [28, 29]. 
Following a standard procedure by diagonalizing Hamiltonian (4.20), we find the Bogolubov spectrum of elementary excitations

$$
\varepsilon_{k}=\sqrt{\omega_{k}^{2}-\Delta_{k}^{2}}
$$

The condition of condensate existence [14, 19] requires that the spectrum should be gapless,

$$
\lim _{k \rightarrow 0} \varepsilon_{k}=0, \quad \varepsilon_{k} \geqslant 0 .
$$

This condition is equivalent to the Hugenholtz-Pines theorem [36]. Hence, we get

$$
\begin{aligned}
\mu_{1}= & \frac{1}{N_{0}} \int \eta^{*}(\mathbf{r})\left\{-\frac{\nabla^{2}}{2 m}+V_{\mathrm{L}}(\mathbf{r})+\Phi_{0}\left[\rho_{0}(\mathbf{r})+2 \rho_{1}(\mathbf{r})\right]\right\} \eta(\mathbf{r}) \mathrm{d} \mathbf{r} \\
& -\frac{\Phi_{0}}{N_{0}} \int \sigma_{1}(\mathbf{r})\left[\eta^{*}(\mathbf{r})\right]^{2} \mathrm{~d} \mathbf{r},
\end{aligned}
$$

where the notations are used for the condensate density

$$
\rho_{0}(\mathbf{r}) \equiv|\eta(\mathbf{r})|^{2},
$$

density of uncondensed atoms

$$
\rho_{1}(\mathbf{r}) \equiv \sum_{k} n_{k}\left|\varphi_{k}(\mathbf{r})\right|^{2}
$$

and the anomalous average

$$
\sigma_{1}(\mathbf{r}) \equiv \sum_{k} \sigma_{k} \varphi_{k}(\mathbf{r}) \varphi_{-k}(\mathbf{r})
$$

The equation for the condensate wave function, in the case of an equilibrium system, is defined by the variational condition

$$
\left\langle\frac{\delta H}{\delta \eta^{*}(\mathbf{r})}\right\rangle=0
$$

which yields the equation

$$
\left\{-\frac{\nabla^{2}}{2 m}+V_{\mathrm{L}}(\mathbf{r})+\Phi_{0}\left[\rho_{0}(\mathbf{r})+2 \rho_{1}(\mathbf{r})\right]\right\} \eta(\mathbf{r})+\Phi_{0} \sigma_{1}(\mathbf{r}) \eta^{*}(\mathbf{r})=\mu_{0} \eta(\mathbf{r}) .
$$

The latter gives the condensate chemical potential

$$
\begin{aligned}
\mu_{0}= & \frac{1}{N_{0}} \int \eta^{*}(\mathbf{r})\left\{-\frac{\nabla^{2}}{2 m}+V_{\mathrm{L}}(\mathbf{r})+\Phi_{0}\left[\rho_{0}(\mathbf{r})+2 \rho_{1}(\mathbf{r})\right]\right\} \eta(\mathbf{r}) \mathrm{d} \mathbf{r} \\
& +\frac{\Phi_{0}}{N_{0}} \int \sigma_{1}(\mathbf{r})\left[\eta^{*}(\mathbf{r})\right]^{2} \mathrm{~d} \mathbf{r} .
\end{aligned}
$$

Comparing expressions 4.23) and (4.29), we see that they are connected by the relation

$$
\mu_{0}=\mu_{1}+\frac{2 \Phi_{0}}{N_{0}} \int \sigma_{1}(\mathbf{r})\left[\eta^{*}(\mathbf{r})\right]^{2} \mathrm{~d} \mathbf{r} .
$$

Evidently, the Lagrange multipliers $\mu_{0}$ and $\mu_{1}$ do not coincide. The system chemical potential is defined through the equation

$$
\langle H\rangle=\langle\hat{H}\rangle-\mu N
$$

which yields

$$
\mu=\frac{1}{N}(\langle\hat{H}\rangle-\langle H\rangle)
$$


This leads to the expression

$$
\mu=\mu_{0} n_{0}+\mu_{1} n_{1}
$$

in which the condensate fraction $n_{0}$ and the fraction of uncondensed atoms, $n_{1}$, are introduced,

$$
n_{0} \equiv \frac{N_{0}}{N}, \quad n_{1} \equiv \frac{N_{1}}{N} .
$$

Invoking equation (4.30), we get

$$
\mu=\mu_{1}+\frac{2 \Phi_{0}}{N} \int \sigma_{1}(\mathbf{r})\left[\eta^{*}(\mathbf{r})\right]^{2} \mathrm{~d} \mathbf{r} .
$$

Sometimes, one requires that $\mu$ should be equal to $\mu_{0}$ and $\mu_{1}$, which forces us to assume that the anomalous average $\sigma_{1}$ should be zero. Such a requirement has no physical reason. In addition, it can be shown by direct calculations [14, 19, 37] that the anomalous average is always comparable with or larger than either the density of uncondensed atoms or that of condensed atoms. Therefore, there is no such a region of parameters, where it could be admissible to neglect the anomalous average, but to keep the normal density and the density of condensed atoms. The sole possibility could be at temperatures close to zero and asymptotically weak interactions, when, though the anomalous average is three times larger than the normal density, both of them are much smaller than the condensate density. Then, it could be possible to omit both the anomalous average and the normal density, keeping only the condensate density. But neglecting one of them, though keeping another one, is mathematically wrong. Moreover, neglecting the anomalous average is not merely mathematically incorrect, but it is qualitatively deficient, making thermodynamics non-self-consistent, disturbing the condensate transition to the first order, and resulting in unphysical divergences of compressibility and structure factor [38].

Since this section is based on the Bloch representation, it is necessary to briefly describe how the Bloch functions could be defined. Formally, as has been mentioned above, the basis of Bloch functions should be chosen as a set of natural orbitals [35], since this gives a diagonal expansion for the density matrix (4.3). However, the problem is that the density matrix (4.2) is not known explicitly. Hence, it is impossible to find its exact eigenfunctions representing the natural orbitals. A standard way is to define the Bloch functions as solutions to the equation

$$
\left[-\frac{\nabla^{2}}{2 m}+V_{\mathrm{L}}(\mathbf{r})\right] \varphi_{n k}(\mathbf{r})=E_{n k} \varphi_{n k}(\mathbf{r}) .
$$

It is also possible to define Bloch functions as eigenfunctions of the nonlinear Schrödinger equation [19], including the interaction term into equation 4.35. Then, calculations become essentially more complicated. In addition, there arises a problem of nonorthogonality of eigenfunctions of the nonlinear equation. Thus, the simplest way is to use the solutions to the linear equation (4.35) as a basis, complimenting it by conservation conditions (4.4).

\section{Wannier representation}

The field operator of atoms can be expanded over the basis of Wannier functions,

$$
\hat{\psi}(\mathbf{r})=\sum_{n j} \hat{c}_{n j} w_{n}\left(\mathbf{r}-\mathbf{a}_{j}\right),
$$

where the index $n=1,2, \ldots$ labels bands and $j=1,2, \ldots, N_{\mathrm{L}}$ enumerates the lattice sites. Substituting this into Hamiltonian (3.2), considering just a single lowest band, and taking into account only the nearestneighbor interactions, one comes to the Hubbard model

$$
\hat{H}=-J \sum_{\langle i j\rangle} \hat{c}_{i}^{\dagger} \hat{c}_{j}+\frac{U}{2} \sum_{j} \hat{c}_{j}^{\dagger} \hat{c}_{j}^{\dagger} \hat{c}_{j} \hat{c}_{j}+h_{0} \sum_{j} \hat{c}_{j}^{\dagger} \hat{c}_{j},
$$


here, the operators $\hat{c}_{j}$ satisfy the Bose commutation relations.

The parameters entering the Hubbard Hamiltonian (5.2) can be calculated in the tight-binding approximation. A detailed demonstration of this calculation can be found in reference [19]. For a threedimensional space in this approximation, we find the expressions

$$
\begin{gathered}
J=\frac{3}{4}\left(\pi^{2}-4\right) V_{0} \exp \left(-\frac{3 \pi^{2}}{4} \sqrt{\frac{V_{0}}{E_{\mathrm{R}}}}\right), \quad U=\sqrt{\frac{8}{\pi}} k_{0} a_{\mathrm{s}} E_{\mathrm{R}}\left(\frac{V_{0}}{E_{\mathrm{R}}}\right)^{3 / 4}, \\
h_{0}=3 E_{\mathrm{R}} \sqrt{\frac{V_{0}}{E_{\mathrm{R}}}} \quad(d=3) .
\end{gathered}
$$

The explanation of the notations for $V_{0}, E_{\mathrm{R}}$, and $k_{0}$ are given in section 2 .

The single-band Hamiltonian (5.2) is called the boson Hubbard model. It is possible to generalize this model by taking into account two or more bands [39, 40]. Here, we consider the single-band case, when the system displays Bose-Einstein condensation, though.

Employing the Bogolubov shift (3.3), we have the condensate wave function

$$
\eta(\mathbf{r})=\sqrt{v n_{0}} \sum_{j} w\left(\mathbf{r}-\mathbf{a}_{j}\right)
$$

with $n_{0}=N_{0} / N$, and the operator of uncondensed atoms

$$
\psi_{1}(\mathbf{r})=\sum_{j} c_{j} w\left(\mathbf{r}-\mathbf{a}_{j}\right)
$$

In terms of the operators $c_{j}$, the Bogolubov shift reads as follows:

$$
\hat{c}_{j}=\sqrt{v n_{0}}+c_{j}
$$

Condition (3.6) leads to the requirement

$$
\left\langle c_{j}\right\rangle=0 .
$$

And from the orthogonality condition [3.7), it follows that

$$
\sum_{j} c_{j}=0
$$

The grand Hamiltonian 3.10, with

$$
\hat{\Lambda}=\sum_{j}\left(\lambda_{j} c_{j}^{\dagger}+\lambda_{j}^{*} c_{j}\right)
$$

takes the form (4.5). The constant $h_{0}$ can be incorporated into the chemical potentials $\mu_{0}$ and $\mu_{1}$. The zero-order term is

$$
H^{(0)}=-J z_{0} n_{0} N+\frac{U}{2} v n_{0}^{2} N-\mu_{0} n_{0} N
$$

where the number of the nearest neighbors is denoted as

$$
z_{0} \equiv \frac{1}{N} \sum_{\langle i j\rangle} 1
$$

The first-order term is canceled by the linear canceler $\hat{\Lambda}$. The second-order term is

$$
H^{(2)}=-J \sum_{\langle i j\rangle} c_{i}^{\dagger} c_{j}+\left(2 U v n_{0}-\mu_{1}\right) \sum_{j} c_{j}^{\dagger} c_{j}+\frac{U}{2} v n_{0} \sum_{j}\left(c_{j}^{\dagger} c_{j}^{\dagger}+c_{j} c_{j}\right) .
$$

The third-order term reads as follows:

$$
H^{(3)}=U \sqrt{v n_{0}} \sum_{j}\left(c_{j}^{\dagger} c_{j}^{\dagger} c_{j}+c_{j}^{\dagger} c_{j} c_{j}\right)
$$


The fourth-order terms is

$$
H^{(4)}=\frac{U}{2} \sum_{j} c_{j}^{\dagger} c_{j}^{\dagger} c_{j} c_{j}
$$

The fraction of uncondensed atoms takes the form

$$
n_{1}=\frac{1}{N} \sum_{j}\left\langle c_{j}^{\dagger} c_{j}\right\rangle=\frac{1}{v}\left\langle c_{j}^{\dagger} c_{j}\right\rangle
$$

where the lattice ideality is used. For the dimensionless anomalous average, we have

$$
\sigma=\frac{1}{N} \sum_{j}\left\langle c_{j} c_{j}\right\rangle=\frac{1}{v}\left\langle c_{j} c_{j}\right\rangle .
$$

The necessary condition of the system stability

$$
\left\langle\frac{\partial H}{\partial N_{0}}\right\rangle=0
$$

yields

$$
\mu_{0}=-J z_{0}+v U\left[n_{0}+2 n_{1}+\frac{1}{2}\left(\sigma^{*}+\sigma\right)\right]+\frac{U}{2 \sqrt{v n_{0}}} \sum_{j}\left\langle c_{j}^{\dagger} c_{j}^{\dagger} c_{j}+c_{j}^{\dagger} c_{j} c_{j}\right\rangle
$$

The operators $c_{j}$ can be expanded over the Fourier basis,

$$
c_{j}=\frac{1}{\sqrt{N_{\mathrm{L}}}} \sum_{k} a_{k} \mathrm{e}^{\mathbf{i k} \cdot \mathbf{a}_{j}},
$$

where $k$ runs over the Brillouin zone.

Let us consider a cubic lattice. Then, the second-order term (5.11) becomes

$$
H^{(2)}=\sum_{k}\left[-2 J \sum_{\alpha=1}^{d} \cos \left(k_{\alpha} a\right)+2 U v n_{0}-\mu_{1}\right] a_{k}^{\dagger} a_{k}+\frac{U}{2} v n_{0} \sum_{k}\left(a_{k}^{\dagger} a_{-k}^{\dagger}+a_{-k} a_{k}\right) .
$$

The third-order and fourth-order terms are

$$
H^{(3)}=U \sqrt{\frac{v n_{0}}{N_{\mathrm{L}}}} \sum_{k p}\left(a_{k}^{\dagger} a_{p}^{\dagger} a_{k+p}+a_{k+p}^{\dagger} a_{p} a_{k}\right)
$$

and, respectively,

$$
H^{(4)}=\frac{U}{2 N_{\mathrm{L}}} \sum_{k p q} a_{k}^{\dagger} a_{p}^{\dagger} a_{k+p} a_{p-q} .
$$

In the HFB approximation, the third-order term is zero, due to condition (5.8). And the fourth-order term in the HFB approximation reads as follows:

$$
H^{(4)}=\frac{v}{2} U \sum_{k}\left(4 n_{1} a_{k}^{\dagger} a_{k}+\sigma a_{k}^{\dagger} a_{-k}^{\dagger}+\sigma^{*} a_{-k} a_{k}\right)-\frac{v}{2} U N\left(2 n_{1}^{2}+|\sigma|^{2}\right) .
$$

Introducing the notations

$$
\omega_{k} \equiv-2 J \sum_{\alpha=1}^{d} \cos \left(k_{\alpha} a\right)+2 v U-\mu_{1}
$$

and

$$
\Delta \equiv v U\left(n_{0}+\sigma\right)
$$


for the grand Hamiltonian 4.5, we obtain

$$
H=E_{\mathrm{HFB}}+\sum_{k} \omega_{k} a_{k}^{\dagger} a_{k}+\frac{1}{2} \sum_{k}\left(\Delta a_{k}^{\dagger} a_{-k}^{\dagger}+\Delta^{*} a_{-k} a_{k}\right),
$$

where

$$
E_{\mathrm{HFB}} \equiv H^{(0)}-v N \frac{U}{2}\left(2 n_{1}^{2}+|\sigma|^{2}\right)
$$

The condensate chemical potential (5.17) in the HFB approximation becomes

$$
\mu_{0}=-z_{0} J+v U\left(1+n_{1}+\sigma\right) .
$$

Diagonalizing Hamiltonian (5.25), we get the Bogolubov Hamiltonian

$$
H_{\mathrm{B}}=E_{\mathrm{B}}+\sum_{k} \varepsilon_{k} b_{k}^{\dagger} b_{k},
$$

in which

$$
E_{\mathrm{B}}=E_{\mathrm{HFB}}+\frac{1}{2} \sum_{k}\left(\varepsilon_{k}-\omega_{k}\right)
$$

and the Bogolubov spectrum is

$$
\varepsilon_{k}=\sqrt{\omega_{k}^{2}-\Delta^{2}}
$$

The condition of the condensate existence (4.22) yields

$$
\mu_{1}=-z_{0} J+v U\left(1+n_{1}-\sigma\right) .
$$

Then, equation (5.23) becomes

$$
\omega_{k}=\Delta+4 J \sum_{\alpha=1}^{d} \sin ^{2}\left(\frac{k_{\alpha} a}{2}\right)
$$

And, introducing the notation

$$
e_{k}=4 J \sum_{\alpha=1}^{d} \sin ^{2}\left(\frac{k_{\alpha} a}{2}\right)
$$

for the Bogolubov spectrum (5.28), we get

$$
\varepsilon_{k}=\sqrt{e_{k}\left(e_{k}+2 \Delta\right)} .
$$

Comparing equations (5.26) and (5.29) yields the relation

$$
\mu_{0}=\mu_{1}+2 v U \sigma .
$$

As is seen, $\mu_{0}$ does not coincide with $\mu_{1}$, by analogy with relation (4.30). The anomalous average cannot be neglected, as is explained in section 4 .

For the quasi-momentum atomic distribution and for the quasi-momentum representation of the anomalous average, respectively, we find

$$
n_{k} \equiv\left\langle a_{k}^{\dagger} a_{k}\right\rangle=\frac{\omega_{k}}{2 \varepsilon_{k}} \operatorname{coth}\left(\frac{\varepsilon_{k}}{2 T}\right)-\frac{1}{2}, \quad \sigma_{k} \equiv\left\langle a_{k} a_{-k}\right\rangle=-\frac{\Delta}{2 \varepsilon_{k}} \operatorname{coth}\left(\frac{\varepsilon_{k}}{2 T}\right) .
$$

This shows that the normal and anomalous averages are connected by the relation

$$
\sigma_{k}^{2}=n_{k}\left(1+n_{k}\right)-\frac{1}{4 \sinh ^{2}\left(\varepsilon_{k} / 2 T\right)} .
$$


For the integral quantities (5.14) and (5.15), we have

$$
n_{1}=\frac{1}{\rho} \int_{\mathscr{B}} n_{k} \frac{\mathrm{d} \mathbf{k}}{(2 \pi)^{d}}, \quad \sigma=\frac{1}{\rho} \int_{\mathscr{B}} \sigma_{k} \frac{\mathrm{d} \mathbf{k}}{(2 \pi)^{d}} .
$$

The condensate fraction reads as

$$
n_{0}=1-\frac{1}{2 \rho} \int_{\mathscr{B}}\left[\frac{\omega_{k}}{\varepsilon_{k}} \operatorname{coth}\left(\frac{\varepsilon_{k}}{2 T}\right)-1\right] \frac{\mathrm{d} \mathbf{k}}{(2 \pi)^{d}},
$$

with the integration over the Brillouin zone.

Let us emphasize again that the anomalous average cannot be neglected for the principal reason. As is evident form the above formulas, the anomalous average can be zero only when there is no condensate, $n_{0}=0$. Hence, there is no gauge symmetry breaking. However, as soon as there appears Bose-Einstein condensate, the gauge symmetry becomes broken, and the anomalous average is never zero. It is always comparable with or larger than either the density of uncondensed atoms or that of condensed atoms.

\section{Thermodynamic characteristics}

In the HFB approximation, the grand potential takes the form

$$
\Omega=E_{\mathrm{B}}+T V \int_{\mathscr{B}} \ln \left(1-\mathrm{e}^{-\beta \varepsilon_{k}}\right) \frac{\mathrm{d} \mathbf{k}}{(2 \pi)^{d}},
$$

where the integration is over the Brillouin zone and

$$
E_{\mathrm{B}}=H^{(0)}-\frac{N}{2} v U\left(2 n_{1}^{2}+\sigma^{2}\right)+\frac{N}{2 \rho} \int_{\mathscr{B}}\left(\varepsilon_{k}-\omega_{k}\right) \frac{\mathrm{d} \mathbf{k}}{(2 \pi)^{d}} .
$$

The system chemical potential 4.33) is

$$
\mu=\mu_{0} n_{0}+\mu_{1} n_{1}=-z_{0} J+v U\left(1+n_{1}+\sigma-2 n_{1} \sigma\right)
$$

For the ground-state energy

$$
E_{0} \equiv E_{\mathrm{B}}+\mu N
$$

we have

$$
\frac{E_{0}}{N}=-z_{0} J+\frac{1}{2} v U\left(1+n_{1}^{2}-\sigma^{2}-2 n_{1} \sigma\right)+\frac{1}{2 \rho} \int_{\mathscr{B}}\left(\varepsilon_{k}-\omega_{k}\right) \frac{\mathrm{d} \mathbf{k}}{(2 \pi)^{d}} .
$$

Atomic fluctuations are characterized by the number-of-atom operator variance

$$
\operatorname{var}(\hat{N}) \equiv\left\langle\hat{N}^{2}\right\rangle-\langle\hat{N}\rangle^{2}
$$

in which

$$
\hat{N}=N_{0}+\hat{N}_{1}
$$

is the operator of the total number of atoms. Since the first term $N_{0}$ is a non-operator number, one has

$$
\operatorname{var}(\hat{N})=\operatorname{var}\left(\hat{N}_{1}\right) \text {. }
$$

In the HFB approximation, we get

$$
\operatorname{var}\left(\hat{N}_{1}\right)=\frac{N T}{v U\left(n_{0}+\sigma\right)} .
$$


The number-of-atom operator variance defines the isothermic compressibility

$$
\kappa_{T}=\frac{\operatorname{var}(\hat{N})}{\rho T N}=\frac{1}{\rho v U\left(n_{0}+\sigma\right)} .
$$

The atomic fluctuations are, of course, normal and the compressibility is finite everywhere below $T_{\mathrm{c}}$. The compressibility can diverge only at the critical point $T_{\mathrm{c}}$.

Bose-Einstein condensation is a second-order phase transition occurring at a temperature $T_{\mathrm{c}}$, where $n_{0}=0$ and $\sigma=0$. At this point, the atomic density is

$$
\rho=\frac{1}{2} \int_{\mathscr{B}}\left[\operatorname{coth}\left(\frac{\omega_{k}}{2 T_{\mathrm{c}}}\right)-1\right] \frac{\mathrm{d} \mathbf{k}}{(2 \pi)^{d}} .
$$

Solving this equation in the Debye approximation, we obtain the critical temperature

$$
T_{\mathrm{c}}=4 \pi \frac{d-2}{d}\left[\Gamma\left(1+\frac{d}{2}\right)\right]^{2 / d} J v .
$$

This tells us that $T_{\mathrm{c}}$ is not defined for $d=1$ and $T_{\mathrm{c}}=0$ for $d=2$. In three dimensions, we have

$$
T_{\mathrm{c}} \simeq 5 \mathrm{Jv} \quad(d=3) .
$$

The general equation for the superfluid fraction [14, 19] can be written in the form

$$
n_{\mathrm{s}}=1-\frac{Q}{Q_{0}}
$$

with the classical dissipated heat

$$
Q_{0} \equiv \frac{d}{2} T
$$

where $d$ is spatial dimensionality, and

$$
Q=\frac{\operatorname{var}(\hat{P})}{2 m N}
$$

is the actual dissipated heat, expressed through the variance of the momentum operator

$$
\hat{\mathbf{P}} \equiv \int \psi_{1}^{\dagger}(\mathbf{r})(-\mathrm{i} \vec{\nabla}) \psi_{1}(\mathbf{r}) \mathrm{d} \mathbf{r}
$$

In an equilibrium system, this variance is

$$
\operatorname{var}(\hat{\mathbf{P}})=\left\langle\hat{\mathbf{P}}^{2}\right\rangle
$$

Note that the condensed fraction does not contribute to the operator of momentum 6.16 due to the lattice periodicity [19].

For a three-dimensional cubic lattice, with a lattice spacing $a$, we obtain

$$
Q=\frac{|\mathbf{p}(\mathbf{a})|^{2}}{2 m \rho} \int_{\mathscr{B}} \frac{\sum_{\alpha} \sin ^{2}\left(k_{\alpha} a\right)}{\sinh ^{2}\left(\varepsilon_{k} / 2 T\right)} \frac{\mathrm{d} \mathbf{k}}{(2 \pi)^{3}},
$$

where the expression

$$
|\mathbf{p}(\mathbf{a})|^{2} \equiv \frac{1}{a^{2}} \exp \left(-\frac{a^{2}}{2 l_{0}^{2}}\right)
$$


is used, derived in the tight-binding approximation. Here, the notation

$$
l_{0} \equiv \frac{1}{\sqrt{m \omega_{0}}}=\frac{1}{\sqrt{2 m}\left(E_{\mathrm{R}} V_{0}\right)^{1 / 4}}
$$

means an effective localization length.

For a three-dimensional cubic lattice, the relations

$$
a^{2}=\frac{3 \pi}{2 m E_{\mathrm{R}}}, \quad k_{0}^{2}=3\left(\frac{\pi}{a}\right)^{2}
$$

are valid, which yield the ratio

$$
\frac{a^{2}}{l_{0}^{2}}=3 \pi^{2} \sqrt{\frac{V_{0}}{E_{\mathrm{R}}}} .
$$

Then, equation (6.19) can be written as follows:

$$
|\mathbf{p}(\mathbf{a})|^{2}=\frac{1}{a^{2}} \exp \left(-\frac{3 \pi^{2}}{2} \sqrt{\frac{V_{0}}{E_{\mathrm{R}}}}\right) .
$$

Comparing this with the tunneling parameter defined in equations (5.3), we have

$$
|\mathbf{p}(\mathbf{a})|^{2}=\frac{1}{2 \pi^{2}}\left(\frac{J}{a V_{0}}\right)^{2} .
$$

Therefore, the dissipated heat 6.18 is written as follows:

$$
Q=\frac{a}{m v}\left(\frac{J}{2 \pi V_{0}}\right)^{2} \int_{\mathscr{B}} \frac{\sum_{\alpha} \sin ^{2}\left(k_{\alpha} a\right)}{\sinh ^{2}\left(\varepsilon_{k} / 2 T\right)} \frac{\mathrm{d} \mathbf{k}}{(2 \pi)^{3}} .
$$

In this way, the self-consistent mean-field approximation allows us to calculate any thermodynamic characteristic.

\section{Conclusion}

A self-consistent approach, based on the use of a representative statistical ensemble, developed earlier for uniform Bose-condensed systems, is extended to Bose atoms in optical lattices. The approach ensures a gapless spectrum of collective excitations, the validity of conservation laws, and self-consistent thermodynamics. It is shown that the approach can be applied to the lattices with a weak binding as well as with tight binding. For the former case, the Bloch representation is more appropriate, while for the latter case, the Wannier representation is more suitable. Both the Bloch and the Wannier representations lead to a similar description. The results are compared for the self-consistent Hartree-Fock-Bogolubov approximation. A convenient general formula for the superfluid fraction of atoms in an optical lattice is derived.

The HFB approximation, used here, is based on the assumption of the condensate existence, which is taken into account by means of the Bogolubov shift, explicitly breaking the global gauge symmetry of the system. This approximation, therefore, is assumed to provide good description, when the Bose condensate is present, and may be inappropriate when the system passes to an insulating state. This implies that the HFB approximation for optical lattices can provide an accurate description for spatial dimensions larger than one $(d>1)$ and nonzero temperatures below the Bose-Einstein condensation temperature, $0<T<T_{\mathrm{c}}$.

The case of zero temperature requires a special consideration. Cubic optical lattices at zero temperature and unity filling factor $v=1$ have been extensively studied, mainly from the viewpoint of an insulating state, with the purpose of defining the stability boundary of this state, corresponding to the critical transition to the superfluid state. The dimensionless parameter

$$
u \equiv \frac{U}{z_{0} J}
$$


has been varied. For a cubic lattice, the number of nearest neighbors is $z_{0}=2 d$. This zero-temperature problem has been treated in the Gutzwiller approximation [41, 42], dynamical mean-field approximation [43], direct numerical diagonalization [44], density-matrix renormalization group [45], strong-coupling perturbation theory [46, 47], and Monte Carlo simulations [48-50]. The critical values of the above parameter were found for $d=1$ as $u_{\mathrm{c}}=1.8$, for $d=2$, as $u_{\mathrm{c}}=4.2$, and for $d=3$, as $u_{\mathrm{c}}=4.9$. The HFB approximation underestimates quantum fluctuations at zero temperature. That is why it is applicable only for nonzero temperatures, when thermal fluctuations become more important.

The advantage of using the developed approach for Bose-condensed atoms in optical lattices at finite temperatures is its relative simplicity, correct gapless spectrum, the validity of conservation laws, and self-consistent thermodynamics.

\section{Acknowledgement}

The author is grateful to E.P. Yukalova for useful discussions. Financial support from the Russian Foundation for Basic Research is acknowledged.

\section{References}

1. Pitaevskii L., Stringari S., Bose-Einstein Condensation, Clarendon, Oxford, 2003.

2. Lieb E.H., Seiringer R., Solovej J.P., Yngvason J., The Mathematics of the Bose Gas and Its Condensation, Birkhauser, Basel, 2005.

3. Letokhov V., Laser Control of Atoms and Molecules, Oxford University, New York, 2007.

4. Pethick C.J., Smith H., Bose-Einstein Condensation in Dilute Gases, Cambridge University, Cambridge, 2008.

5. Courteille P.W., Bagnato V.S., Yukalov V.I., Laser Phys., 2001, 11, 659.

6. Andersen J.O., Rev. Mod. Phys., 2004, 76, 599; doi 10.1103/RevModPhys.76.599

7. Yukalov V.I., Laser Phys. Lett., 2004, 1, 435; doi 10.1002/lapl.200410097

8. Bongs K., Sengstock K., Rep. Prog. Phys., 2004, 67, 907; doi 10.1088/0034-4885/67/6/R03

9. Yukalov V.I., Girardeau M.D., Laser Phys. Lett., 2005, 2, 375; doi 10.1002/lapl.200510011

10. Posazhennikova A., Rev. Mod. Phys., 2006, 78, 1111; doi 10.1103/RevModPhys.78.1111

11. Yukalov V.I., Laser Phys. Lett., 2007, 4, 632; doi 10.1002/lapl.200710029

12. Proukakis N.P., Jackson B., J. Phys. B, 2008, 41, 203002; doi 10.1088/0953-4075/41/20/203002.

13. Yurovsky V.A., Olshanii M., Weiss D.S., Adv. Atom. Mol. Opt. Phys., 2008, 55, 61; doi 10.1016/S1049-250X(07)55002-0.

14. Yukalov V.I., Phys. Part. Nucl., 2011, 42, 460; doi 10.1134/S1063779611030063

15. Yukalov V.I., Laser Phys., 2012, 22, 1145; doi 10.1134/S1054660X12070171

16. Morsch O., Oberthaler M., Rev. Mod. Phys., 2006, 78, 179; doi 10.1103/RevModPhys.78.179

17. Moseley C., Fialko O., Ziegler K., Ann. Phys. (Berlin), 2008, 17, 561.

18. Bloch I., Dalibard J., Zwerger W., Rev. Mod. Phys., 2008, 80, 885; doi 10.1103/RevModPhys.80.885

19. Yukalov V.I., Laser Phys., 2009, 19, 1; doi 10.1134/S1054660X09010010

20. Hohenberg P.C., Martin P.C., Ann. Phys. (N.Y.), 1965, 34, 291; doi 10.1016/0003-4916(65)90280-0

21. Gibbs J.W., Elementary Principles in Statistical Mechanics, Oxford University, Oxford, 1902.

22. Yukalov V.I., Phys. Rep., 1991, 208, 395; doi 10.1016/0370-1573(91)90074-V

23. Yukalov V.I., Phys. Rev. E, 2005, 72, 066119; doi 10.1103/PhysRevE.72.066119

24. Yukalov V.I., Phys. Lett. A, 2006, 359, 712; doi 10.1016/j.physleta.2006.07.060

25. Yukalov V.I., Laser Phys., 2006, 16, 511; doi 10.1134/S1054660X06030145

26. Yukalov V.I, Int. J. Mod. Phys. B, 2007, 21, 69; doi 10.1142/S0217979207035893

27. Yukalov V.I., Ann. Phys. (N.Y.), 2008, 323, 461; doi 10.1016/j.aop.2007.05.003

28. Bogolubov N.N., Lectures on Quantum Statistics, Vol. 1, Gordon and Breach, New York, 1967.

29. Bogolubov N.N., Lectures on Quantum Statistics, Vol. 2, Gordon and Breach, New York, 1970.

30. Yukalov V.I., Kleinert H., Phys. Rev. A, 2006, 73, 063612; doi 10.1103/PhysRevA.73.063612

31. Yukalov V.I., Yukalova E.P., Phys. Rev. A, 2006, 74, 063623; doi 10.1103/PhysRevA.74.063623.

32. Yukalov V.I., Yukalova E.P., Phys. Rev. A, 2007, 76, 013602; doi $10.1103 /$ PhysRevA.76.013602.

33. Yukalov V.I., Graham R., Phys. Rev. A, 2007, 75, 023619; doi 10.1103/PhysRevA.75.023619

34. Yukalov V.I., Yukalova E.P., Krutitsky K.V., Graham R., Phys. Rev. A, 2007, 76, 053623; doi 10.1103/PhysRevA.76.053623 
35. Coleman A.J., Yukalov V.I., Reduced Density Matrices, Springer, Berlin, 2000.

36. Hugenholtz N.M., Pines D., Phys. Rev., 1959, 116, 489; doi 10.1103/PhysRev.116.489

37. Yukalov V.I., Yukalova E.P., Laser Phys. Lett., 2005, 2, 506; doi 10.1002/lapl.200510031

38. Yukalov V.I., J. Phys. Stud., 2007, 11, 55.

39. Yukalov V.I., Rakhimov A., Mardonov S., Laser Phys., 2011, 21, 264; doi 10.1134/S1054660X11010233

40. Stasyuk I.V., Velychko O.V., Condens. Matter Phys., 2012, 15, 33002; doi 10.5488/CMP.15.33002

41. Rokhsar D.S., Kotliar B.G., Phys. Rev. B, 1991, 44, 10328; doi 10.1103/PhysRevB.44.10328

42. Schroll C., Marquardt F., Bruder C., Phys. Rev. A, 2004, 70, 053609; doi 10.1103/PhysRevA.70.053609

43. Amico L., Penna V., Phys. Rev. Lett., 1998, 80, 2189; doi 10.1103/PhysRevLett.80.2189

44. Roth R., Burnett K., Phys. Rev. A, 2003, 67, 031602; doi 10.1103/PhysRevA.67.031602

45. Kühner T.D., White S.R., Monien H., Phys. Rev. B, 2000, 61, 12474; doi PhysRevB.61.12474

46. Elstner N., Monien H., Phys. Rev. B, 1999, 59, 12184; doi 10.1103/PhysRevB.59.12184

47. Damski B., Zakrzewski J., Phys. Rev. A, 2006, 74, 043609; doi 10.1103/PhysRevA.74.043609

48. Wessel S., Alet F., Troyer M., Batrouni G.G., Phys. Rev. A, 2004, 70, 053615; doi 10.1103/PhysRevA.70.053615

49. Capogrosso-Sansone B., Prokofiev N.V., Svistunov B.V., Phys. Rev. B, 2007, 75, 134302; doi 10.1103/PhysRevB.75.134302

50. Capogrosso-Sansone B., Söyler S.G., Prokofiev N., Svistunov B., Phys. Rev. A, 2008, 77, 015602; doi 10.1103/PhysRevA.77.015602 


\section{Самоузгоджений метод для атомів Бозе-конденсату в оптичних гратках}

\section{B.I. Юкалов}

Лабораторія теоретичної фізики ім. М.М. Боголюбова, Об'єднаний інститут ядерних досліджень, 141980 Дубна, Росія

Розглядаються атоми Бозе в оптичних гратках при низьких температурах і слабких взаємодіях, коли конденсат Бозе-Ейнштейна є утворений. Застосовано самоузгоджений підхід, що базується на використанні репрезентативного статистичного ансамблю і забезпечує безщілинний спектр колективних збуджень i чинність законів збереження. Для того, щоб показати застосовність підходу до обох, слабкого і сильного зв'язку, проблема розглядається в представленнях Блоха і Ваньє. Обидва способи приводять до подібних виразів, що порівнюються з самоузгодженим наближенням Хартрі-Фока-Боголюбова. Отримано зручну загальну формулу для надплинної фракції атомів в оптичній гратці.

Ключові слова: конденсат Бозе-Ейштейна, репрезентативний ансамбль, оптичні гратки, представлення Блоха, представлення Ваньє, надплинність 